\title{
EPHX1 polymorphisms, COPD and asthma in 47,000 individuals and in meta-analysis
}

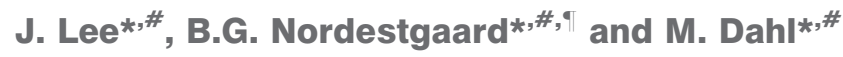

ABSTRACT: We tested the hypothesis that two well-characterised functional polymorphisms of the microsomal epoxide hydrolase gene (EPHX1), T113C and A139G, may influence susceptibility to chronic obstructive pulmonary disease (COPD) and asthma.

We genotyped participants from the Copenhagen City Heart Study $(n=10,038)$ and the Copenhagen General Population Study $(n=37,022)$ for the $T 113 C$ and A139G variants in the EPHX1 gene and measured lung function and recorded COPD hospitalisation and asthma and smoking history. Finally, we meta-analysed results from 19 studies including 7,489 COPD cases and 42,970 controls.

The OR for spirometry-defined COPD or COPD hospitalisation did not differ from 1.0 for any of the EPHX1 genotypes or phenotypes overall, or in smokers or nonsmokers separately ( $p$-value for trend 0.18-0.91). Likewise, EPHX1 genotypes or phenotypes did not associate with risk of asthma ( $p$-value for trend 0.46-0.98). In meta-analysis, random effects OR for COPD in T113C heterozygotes and homozygotes versus non-carriers were 1.17 (0.99-1.38) and 1.38 (1.09-1.74), respectively. Corresponding values for A139G were $0.93(0.83-1.05)$ and 0.89 (0.78-1.02).

Our results indicate that genetically reduced microsomal epoxide hydrolase activity is not a major risk factor for COPD or asthma in the Danish population; however, meta-analysis cannot completely exclude a minor effect on COPD risk.

KEYWORDS: Chronic obstructive pulmonary disease, genetics, lung function, meta-analysis, microsomal epoxide hydrolase, tobacco smoking

hronic obstructive pulmonary disease (COPD) is a complex disease characterised by airflow limitation that is not fully reversible. This is usually progressive and associated with an abnormal inflammatory response of the lungs to noxious particles and gases. The clinical manifestations include chronic bronchitis, emphysema and small airway disease with many patients suffering from all three [1]. Tobacco smoking is widely accepted as the most common risk factor for COPD [1, 2]. However, only a fraction of smokers (10-15\%) develop the disease [3]. This, together with the familial clustering of early-onset COPD [4] as well as susceptibility to frequent exacerbations in these individuals [5], strongly indicates a genetic aspect in the pathogenesis of COPD.

The microsomal epoxide hydrolase gene, EPHX1, is a good candidate for several reasons. First, it is strongly expressed in the lung, but downregulated in COPD [6]. Secondly, the enzyme product has an established role in the detoxification of smoking-induced reactive substances which may cause oxidative stress [7]. Finally, there are two well-characterised variants of the EPHX1 gene which have been shown to alter enzyme activity considerably [8]. This might account for some of the variety in susceptibility to COPD among smokers.

In the present study, we used data from the Copenhagen City Heart Study, Copenhagen, Denmark $(n=10,038)$ and the Copenhagen General Population Study, Copenhagen, Denmark ( $n=$ $37,022)$ to test the hypotheses that genetically altered microsomal epoxide hydrolase activity is associated with risk of COPD and that this relationship could depend on smoking history. Since asthma is another manifestation of chronic pulmonary inflammation, we also tested for association with risk of asthma. Finally, we meta-analysed present and previous studies on risk of COPD.

\section{MATERIAL AND METHODS}

\section{Study cohort}

In this population-based cross-sectional study, we studied randomly selected white individuals of Danish descent $(n=47,060)$ consisting of participants from two very similar cohorts, the
AFFILIATIONS

*Dept of Clinical Biochemistry, Herlev Hospital, Copenhagen University Hospital, Faculty of Health Sciences, University of Copenhagen, \#The Copenhagen General Population Study, Herlev Hospital, Copenhagen University Hospital, Faculty of Health Sciences, University of Copenhagen, and 'The Copenhagen City Heart Study, Bispebjerg Hospital, Copenhagen University Hospital, Faculty of Health Sciences, University of Copenhagen, Copenhagen, Denmark.

\section{CORRESPONDENCE}

M. Dahl

Dept of Clinical Biochemistry 54M1, Herlev Hospital

Copenhagen University Hospital

Herlev Ringvej 75

DK-2730 Herlev

Denmark

E-mail: mordah02@heh.regionh.dk

Received:

Jan 242010

Accepted after revision:

May 052010

First published online:

June 012010 
Copenhagen City Heart Study $(n=10,038)$ and the Copenhagen General Population Study $(n=37,022)$ [9-11]. In brief, the Copenhagen City Heart Study is a prospective cardiopulmonary study of the Danish general population initiated in 19761978, with follow-up examinations in 1981-1983, 1991-1994 and 2001-2003; DNA was taken in 1991-1994 and 2001-2003. The Copenhagen General Population Study is a cross-sectional study of the Danish general population initiated in 2003 and still recruiting. Individuals were randomly selected based on the national Danish Civil Registration System to reflect the Copenhagen general population aged 20-80 yrs and older. Information on diagnoses of COPD (World Health Organization International Classification of Diseases 8th (ICD-8) and 10th (ICD-10) edition, ICD-8 codes 491-492 and ICD-10 codes J41-J44) was collected in the national Danish Patient Registry and the national Danish Causes of Death Registry. All subjects answered similar questionnaires and had objective and clinical parameters measured by the same methods.

The studies were approved by Herlev Hospital (Copenhagen, Denmark) and Danish ethical committees and were conducted according to the Declaration of Helsinki. Written informed consent was obtained from all participants.

\section{Genotyping}

Taqman assays analysed on the ABI PRISM ${ }_{\circledast} 7900 \mathrm{HT}$ Sequence Detection System (Applied Biosystems Inc., Foster City, CA, USA) were used to genotype two polymorphisms in the EPHX1 gene (T113C (rs1051740) and A139G (rs2234922)). These two single nucleotide polymorphisms (SNPs) were chosen because they are functional and have previously been investigated in relation to the end-points under investigation in the current study. Furthermore, these two SNPs tag the entire coding region of the EPHX1 gene [12]. Since we performed reruns twice, call rates were $99.98 \%$ for both polymorphisms. Genotyping was verified by DNA sequencing (MegaBase, Pharmacia, Uppsala, Sweden).

\section{Other covariates}

Participants filled out a questionnaire stating information about their current and previous diseases and use of medication. Participants were divided based on their smoking history into "never-smokers", "light smokers" (<15 pack-yrs), or "heavy smokers" ( $\geqslant 15$ pack-yrs); 15 was the median of the cumulated pack-yrs.

\section{End-points}

Forced expiratory volume in $1 \mathrm{~s}$ (FEV1) and forced vital capacity (FVC) were determined using a dry-wedge spirometer (Vitalograph, Maids Moreton, Buckinghamshire, UK) in the Copenhagen City Heart Study and using an EasyOne Spirometer (ndd Medizintechnik, Zürich, Switzerland) in the Copenhagen General Population Study. Triplicates with the two best measurements differing by $<5 \%$ were obtained, and the best results were used in the analyses. Reference values were derived for males and females separately in the two studies, based on all participants regardless of smoking history [11]. COPD was defined as FEV1/FVC $<0.7$ and FEV $1<80 \%$ of the predicted value, excluding those with self-reported asthma.

Information about COPD hospitalisation was obtained by linking the participants to the national Danish Patient Registry and the national Danish Causes of Death Registry, using each participant's unique Central Person Register number [9, 10]. The COPD diagnoses were defined according to World Health Organization International Classification of Diseases, 8th and 10th edition (ICD-8: 491-492; ICD-10: J41-J44).

Asthma diagnoses were based on the following three questions. Asthma: "Do you have asthma?"; asthma medication: "Do you take medication for asthma/bronchitis daily or almost daily?"; and allergic asthma: "Do foodstuffs, medicine, grass, flowers, animal hair, or other things give you asthma?".

\section{Statistical analyses}

We used STATA/SE 10.1 (Stata Corporation, College Station, TX, USA) for all statistical analyses, except for the power calculations which were performed with the NCSS/PASS software (NCSS, Kaysville, UT, USA). We used the event rate in a two-sided hypothesis test at $\alpha=0.05$ and $\beta=0.10$ to calculate the minimum effect size that we had $90 \%$ power to detect in our study. For trend tests, individual genotypes were coded 0-2 with homozygosity for the common allele as the reference. Based on the predicted microsomal epoxide hydrolase activity as described by HASSETT et al. [8], participants were divided into four groups encoded 1-4 ("Fast" to "Superslow"; Fast: 113TT/139AG, 113TT/139GG, 113TC/139GG; Normal: 113TT/139AA, 113TC/139AG, 113CC/139GG; Slow: 113TC/ 139AA; Superslow: 113CC/139AG, 113CC/139AA). We chose to analyse these phenotype groupings to maximise statistical power and to simplify data presentation and interpretation; however, if we examined each of the nine genotype combinations separately, results were similar to those presented for the four phenotype groupings.

We first analysed the relationship of EPHX1 genotype and phenotype with FEV1\% predicted. In the subset of individuals from the Copenhagen City Heart Study, we analysed the relationship of EPHX1 genotype and phenotype with decline in FEV1 $\left(\mathrm{mL} \cdot \mathrm{yr}^{-1}\right)$. Analyses were performed by ANOVA adjusted for age, sex and pack-yrs.

We then analysed the relationship between EPHX1 genotype and phenotype and risk of COPD or asthma by logistic regression adjusted for age, sex and pack-yrs.

\section{Meta-analyses}

We searched PubMed and Embase querying for "(COPD [MeSH] OR pulmonary emphysema [MeSH] OR COPD OR chronic obstructive pulmonary disease [All fields]) AND (EPHX1 OR microsomal epoxide hydrolase)" using MeSH/ EMTREE terms and free text. 19 studies (the present study and $[2,7,13-28])$ investigating the EPHX1 T113C and A139G polymorphisms in relation to COPD or emphysema as the primary end-point were included. Two minor studies were excluded from the analyses, because we were unable to retrieve the original papers despite contacting the corresponding authors.

Meta-analyses were performed using random and fixed effect models in STATA/SE 10.1 (Stata Corporation). Weights were calculated under fixed effect models using the inverse variance method. We also calculated summary odds ratios for Caucasians and Asians separately. Funnel plots and Egger's regression test were used to search for publication bias. 


\begin{tabular}{|c|c|c|c|}
\hline \multirow[t]{3}{*}{ TABLE 1} & \multicolumn{3}{|c|}{$\begin{array}{l}\text { Characteristics of participants by chronic } \\
\text { obstructive pulmonary disease (COPD) status }\end{array}$} \\
\hline & \multirow[t]{2}{*}{ No event } & \multicolumn{2}{|c|}{ COPD } \\
\hline & & Spirometry-defined & Hospitalisation \\
\hline Individuals $\mathrm{n}$ & 37964 & 4127 & 2730 \\
\hline Females \% & 54 & 49 & 53 \\
\hline Age yrs & $58(47-68)$ & $68(60-75)$ & $69(62-76)$ \\
\hline \multicolumn{4}{|l|}{ Smoking \% } \\
\hline Never-smokers & 38 & 13 & 6 \\
\hline Light smokers & 34 & 27 & 23 \\
\hline Heavy smokers & 29 & 60 & 71 \\
\hline Pack-yrs & $12(3-27)$ & $26(12-42)$ & $30(15-45)$ \\
\hline
\end{tabular}

Data are presented as median (interquartile range), unless otherwise stated.

\section{RESULTS}

Clinical characteristics of the participants are presented according to COPD status in table 1 . Of the 47,060 participants, 37,964 were free of COPD, 4,127 had COPD according to spirometry and 2,730 were hospitalised due to COPD (967 individuals had both COPD on spirometry and were hospitalised for COPD). As expected, both individuals with spirometry-defined COPD and COPD hospitalisation were older and more likely to be heavy smokers than individuals without COPD. The proportion of females in each group was similar (54\% in individuals without COPD versus $49 \%$ and $53 \%$ for spirometry-defined COPD and COPD hospitalisation, respectively). Of the 47,060 subjects, 33,255 were free of asthma, 3,216 reported asthma, 3,312 reported using asthma medication on a daily or almost daily basis, and 3,754 reported allergic asthma (table 1 in the online supplementary material); there were large overlaps among the three asthma groups. Genotype frequencies were in accordance with the HardyWeinberg equilibrium and corresponded well with those previously reported for Caucasians [7, 13]. The binding of primers and probes was not influenced by the codon119 polymorphism (sequences reported in table 2 in the online

a)

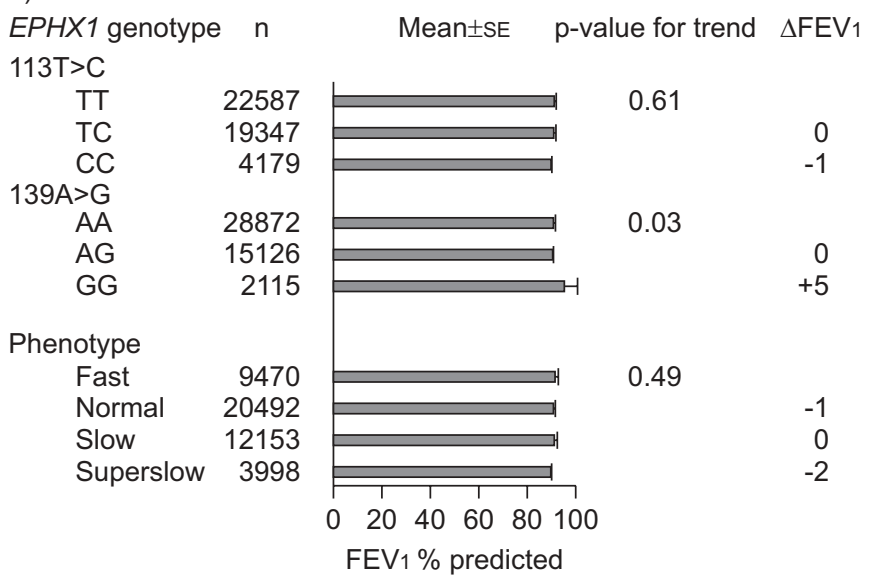

supplementary material) [29]. Since we performed reruns three times, call rates were $99.98 \%$ for both polymorphisms. Control sequencing showed $100 \%$ concordance with the TaqMan genotyping results.

\section{FEV $1 \%$ pred}

The T113C polymorphism and EPHX1 phenotype were not associated with FEV1 \% pred (p-value for trend was 0.61 and 0.49 , respectively; fig. 1a). The $p$-value for trend for the $139 \mathrm{~A}>\mathrm{G}$ polymorphism reached statistical significance $(p=0.03)$; however, this result could not be confirmed when analysing EPHX1 phenotype or other COPD-related traits, and we therefore interpret this as likely a spurious result.

EPHX1 phenotype was not associated with FEV1 \% pred when stratified by smoking status ( $p$-value for trend $0.53-0.83$; fig. 2a).

\section{Decline in lung function}

The observed mean values of lung function decline for the reference genotypes and phenotype were 29-30 $\mathrm{mL} \cdot \mathrm{yr}^{-1}$. EPHX1 genotypes or phenotypes were not associated with decline in lung function in the subset of subjects from the Copenhagen City Heart Study ( $\mathrm{p}$-value for trend 0.64-0.71; fig. 1b).

On visual inspection, there appeared to be a trend towards increased decline in lung function with predicted decreased microsomal epoxide hydrolase activity among heavy smokers, but the trend was not statistically significant ( $\mathrm{p}$-value for trend 0.22 ; fig. $2 b$ ).

\section{Risk of COPD}

The OR for spirometry-defined COPD did not differ from 1.0 for any of the EPHX1 genotypes or phenotypes ( $\mathrm{p}$-value for trend 0.34-0.85; fig. 3). Likewise, OR for hospitalisation due to COPD did not differ from 1.0 for any of the EPHX1 genotypes or phenotypes ( $p$-value for trend 0.24-0.70); we had statistical power to detect a $20-30 \%$ increase in risk of COPD for all individual genotypes and phenotypes overall (figs 2 and 3 in the online supplementary material).

After stratification by smoking status, OR for spirometrydefined COPD and COPD hospitalisation did not differ from 1.0 for any of the EPHX1 phenotypes in either category of

b)

\begin{tabular}{|c|c|c|c|c|}
\hline$E P H X 1$ genotype & $\mathrm{n}$ & Mean \pm SE & $p$-value for trend & $\Delta$ Loss \\
\hline $113 \mathrm{~T}>\mathrm{C}$ & & & & \\
\hline TT & 4133 & 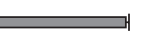 & 0.71 & \\
\hline TC & 3614 & 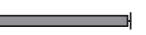 & & +1 \\
\hline CC & 731 & ل्य & & +4 \\
\hline $139 A>G$ & & & & \\
\hline AA & 5309 & 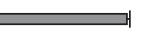 & 0.68 & \\
\hline$A G$ & 2787 & 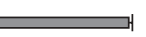 & & 0 \\
\hline GG & 382 & & & 0 \\
\hline henotype & & & & \\
\hline Fast & 1719 & $\Longrightarrow$ & 0.64 & \\
\hline Normal & 3820 & بح & & 0 \\
\hline Slow & 2240 & $\Longrightarrow$ & & 0 \\
\hline Superslow & 699 & $\overline{\mid \bar{T}}$ & & +4 \\
\hline & & $10 \quad 20 \quad 30$ & 40 & \\
\hline
\end{tabular}

FIGURE 1. Levels of a) forced expiratory volume in $1 \mathrm{~s}(\mathrm{FEV} 1) \%$ predicted and b) annual loss in FEV1 according to EPHX1 genotype and enzyme activity phenotype. 
a)

Never-smokers
Fast
Normal
Slow
Superslow
Light smokers
Fast
Normal
Slow
Superslow
Heavy smokers
Fast
Normal
Slow
Superslow

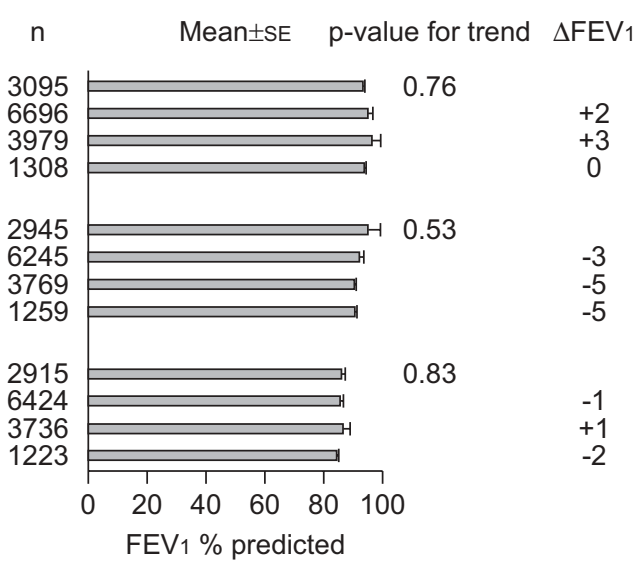

b)

Never-smokers
Fast
Normal
Slow
Superslow
Light smokers
Fast
Normal
Slow
Superslow
Heavy smokers
Fast
Normal
Slow
Superslow

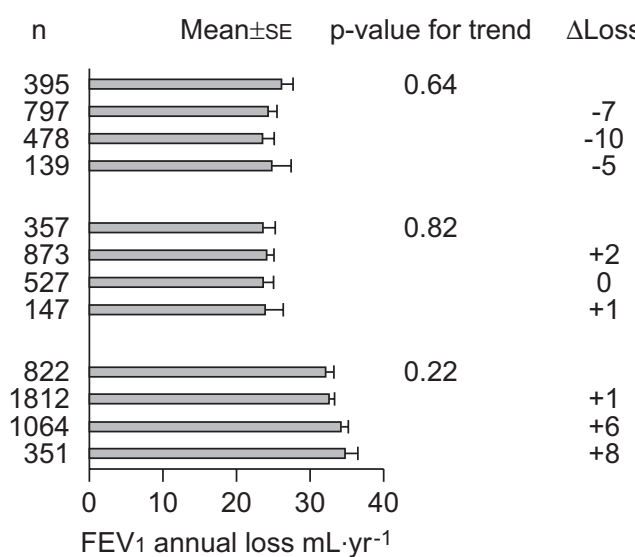

FIGURE 2. Levels of a) forced expiratory volume in $1 \mathrm{~s}\left(F E V_{1}\right) \%$ predicted and b) annual loss in FEV 1 according to EPHX1 enzyme activity phenotype, stratified by smoking history. Light smokers: $<15$ pack-yrs; heavy smokers: $\geqslant 15$ pack-yrs.

smokers (p-value for trend 0.18-0.47; fig. 4). Among neversmokers, the OR for hospitalisation due to COPD did deviate from $1.0(0.60,95 \%$ CI $0.40-0.88)$; however, this result is not biologically plausible in never-smokers, and the finding could not be confirmed when analysing spirometry-defined COPD. Therefore, this finding likely represents a spurious result.

\section{Meta-analyses}

The overall OR (95\% CI) for COPD using random effect models were 1.17 (0.99-1.38) in T113C heterozygotes and 1.38 (1.091.74) in homozygotes versus non-carriers (fig. 5). The corresponding ORs were $0.93(0.83-1.05)$ in A139G heterozygotes and 0.89 (0.78-1.02) in homozygotes (fig. 6). ORs obtained using fixed effect models as well as stratified for ethnicity are also shown in figures 5 and 6 .

\section{Risk of asthma}

The OR for asthma by definition of asthma medication use was increased for the superslow phenotype (1.21, 95\% CI 1.02-1.44; fig. 1 in the online supplementary material). However, this finding was not replicated in the analyses of self-reported asthma or allergic asthma, and it is therefore likely a spurious result. None of the risk estimates for EPHX1 genotypes or other phenotypes differed from 1.0 in any of the three asthma categories ( $p$-value for trend 0.46-0.98, 0.17-0.98, 0.75-0.82, respectively).

\section{DISCUSSION}

With COPD on the rise as a leading cause of morbidity and mortality worldwide, much interest has been focused on uncovering the underlying mechanisms and, thus, potential therapeutic targets. It seems clear that many genetic factors may influence an individual's susceptibility to COPD. A handful of studies have focused on the EPHX1 gene and especially two common polymorphisms, which reportedly alter enzyme activity [8]. Although the involvement of EPHX1 in COPD seems plausible, reports on the effect of these polymorphisms on COPD risk have been inconsistent. Our
Spirometry-defined COPD

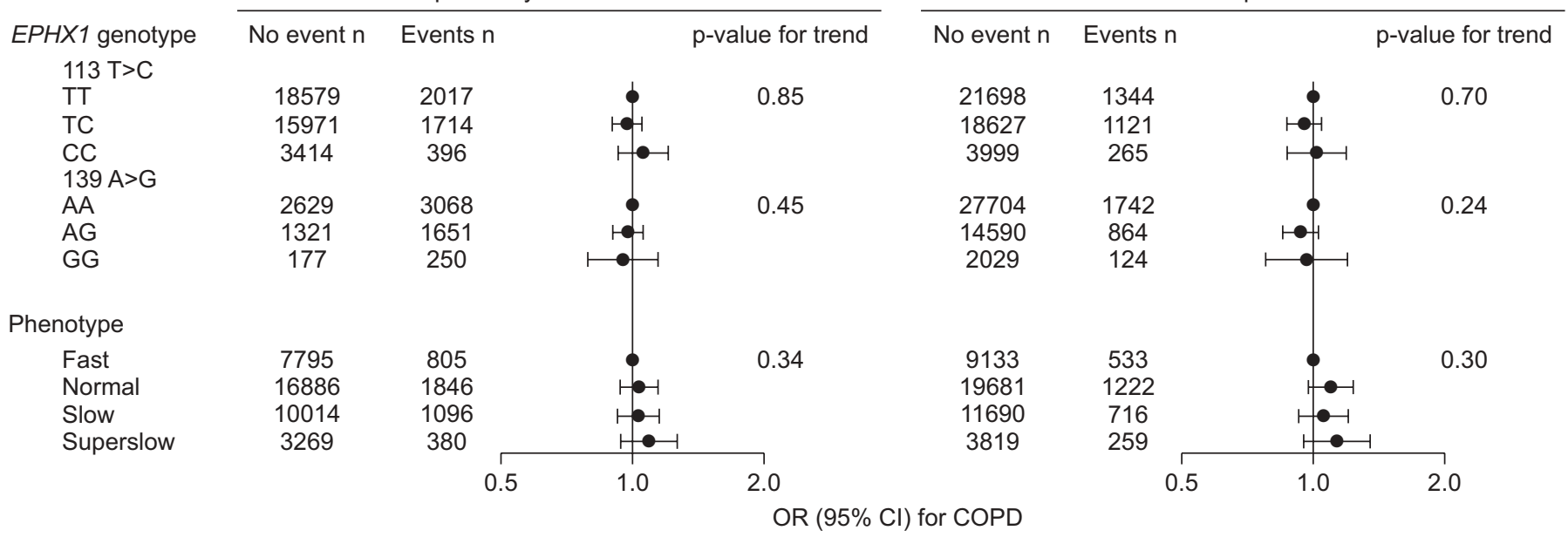

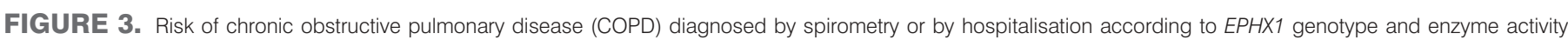

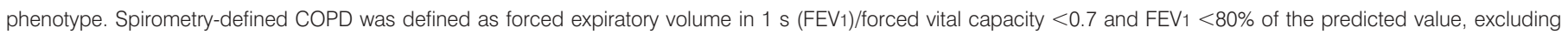

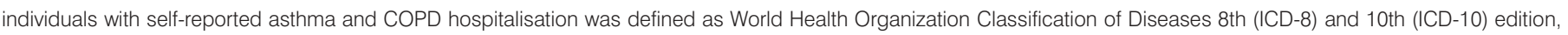
ICD-8: 491-492; ICD-10: J41-J44. Event: individuals with the relevant end-point; no event: disease-free controls by any of the definitions investigated. 
Spirometry-defined COPD

$\begin{array}{lcc}\text { Never-smokers } & & \\ \text { Fast } & 2776 & 95 \\ \text { Medium } & 6031 & 239 \\ \text { Slow } & 3579 & 141 \\ \text { Superslow } & 1181 & 48 \\ \text { Light smokers } & & \\ \text { Fast } & 2494 & 209 \\ \text { Medium } & 5270 & 479 \\ \text { Slow } & 3199 & 271 \\ \text { Superslow } & 1054 & 103 \\ \text { Heavy smokers } & & \\ \text { Fast } & 2099 & 461 \\ \text { Medium } & 4621 & 1044 \\ \text { Slow } & 2665 & 636 \\ \text { Superslow } & 866 & 205\end{array}$

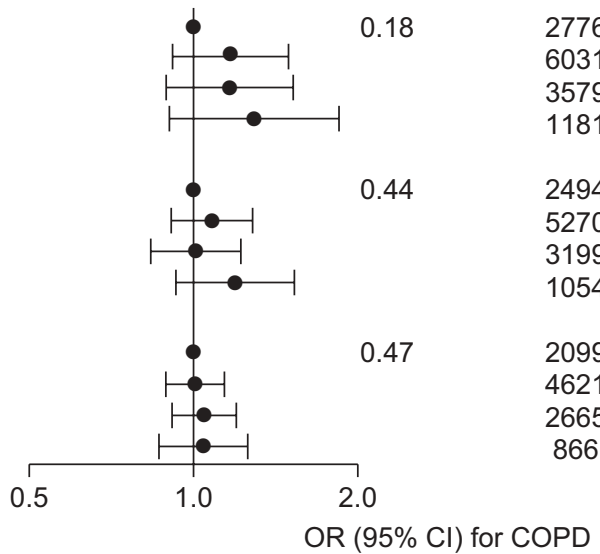

COPD hospitalisation

No event $n$ Events $n \quad p$-value for trend

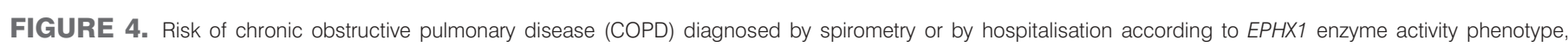

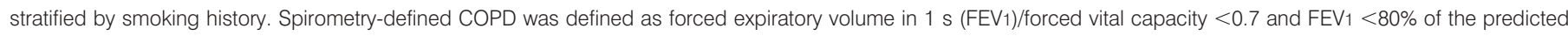

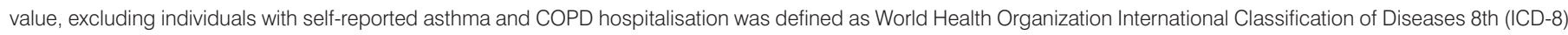

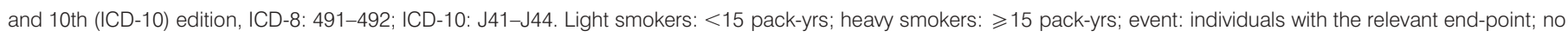
event: disease-free controls by any of the definitions investigated.

study contributes results from the hitherto largest populationbased investigation of EPHX1 as a risk factor in COPD. Since we had statistical power to detect even a $20-30 \%$ increased risk of COPD for all EPHX1 genotypes and phenotypes overall, and since we previously found positive associations between risk alleles in other genes and risk of COPD using the same cohorts

\section{EPHX1 T113C}

First author [ref.] PARK [14] YIM [15] TAKEYABU [16]

XIAO [17]

RodRIGUEZ [18]

BUDHI [19]

KORYTINA [20]

SMITH [21]

MATHESON [22]

VIBHUTI [23]

ZIDZIK [24]

PARK [25]

CHENG [26]

BRøGGER [2]

Fu [27]

HERSH [28]

SIEDLINSKI [13]

Current study

Random effects

Overall

Caucasian

Asian

Fixed effects

Overall

Caucasian

Asian
Chappell [7]

Heterozygotes

Homozygotes

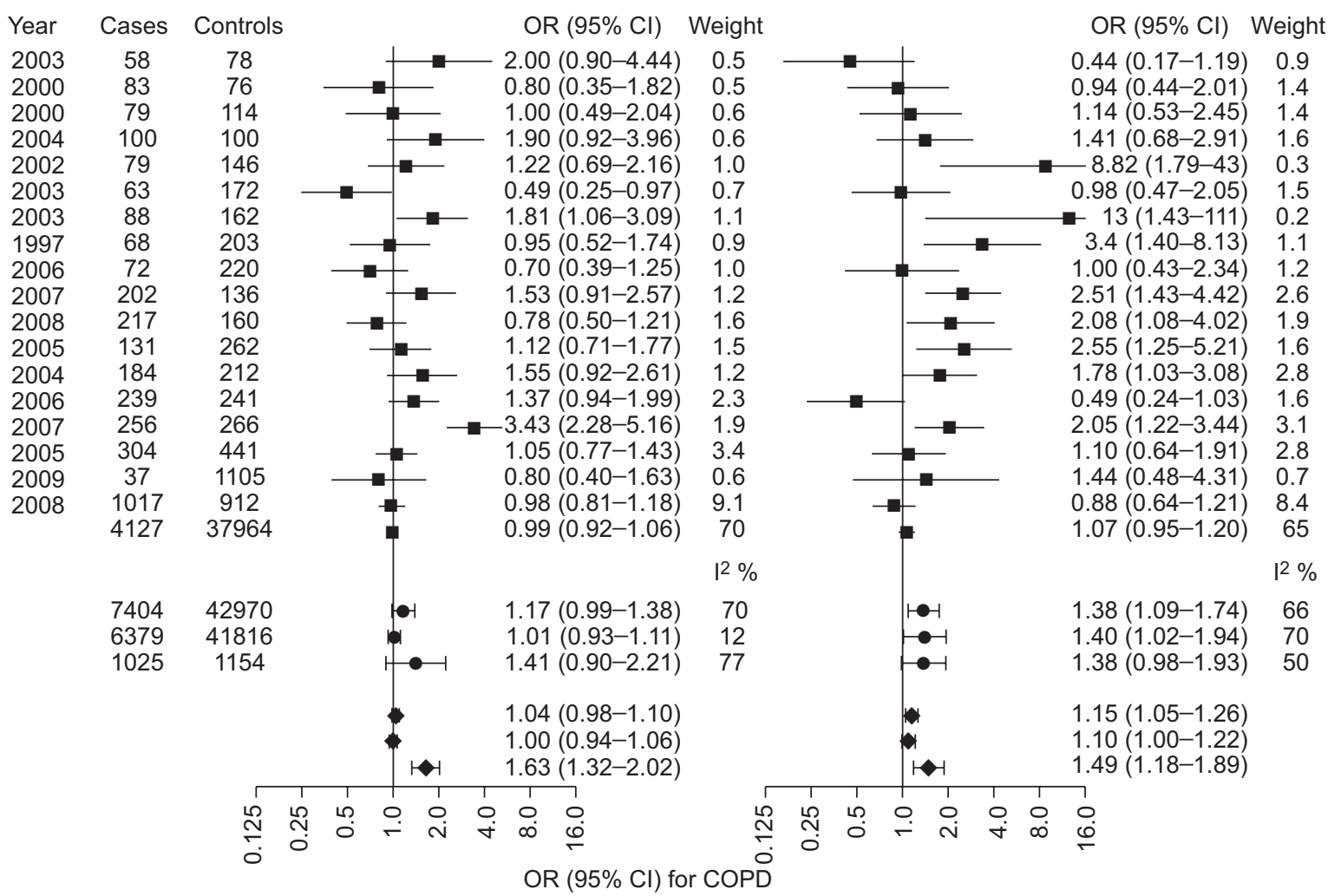

FIGURE 5. Meta-analysis of EPHX1 T113C genotype and risk of chronic obstructive pulmonary disease (COPD); overall and stratified analyses using both random and fixed effect models. Weights are from the fixed effect analyses using the inverse variance method. $\mathrm{I}^{2}$ : the variation in OR attributable to heterogeneity. 


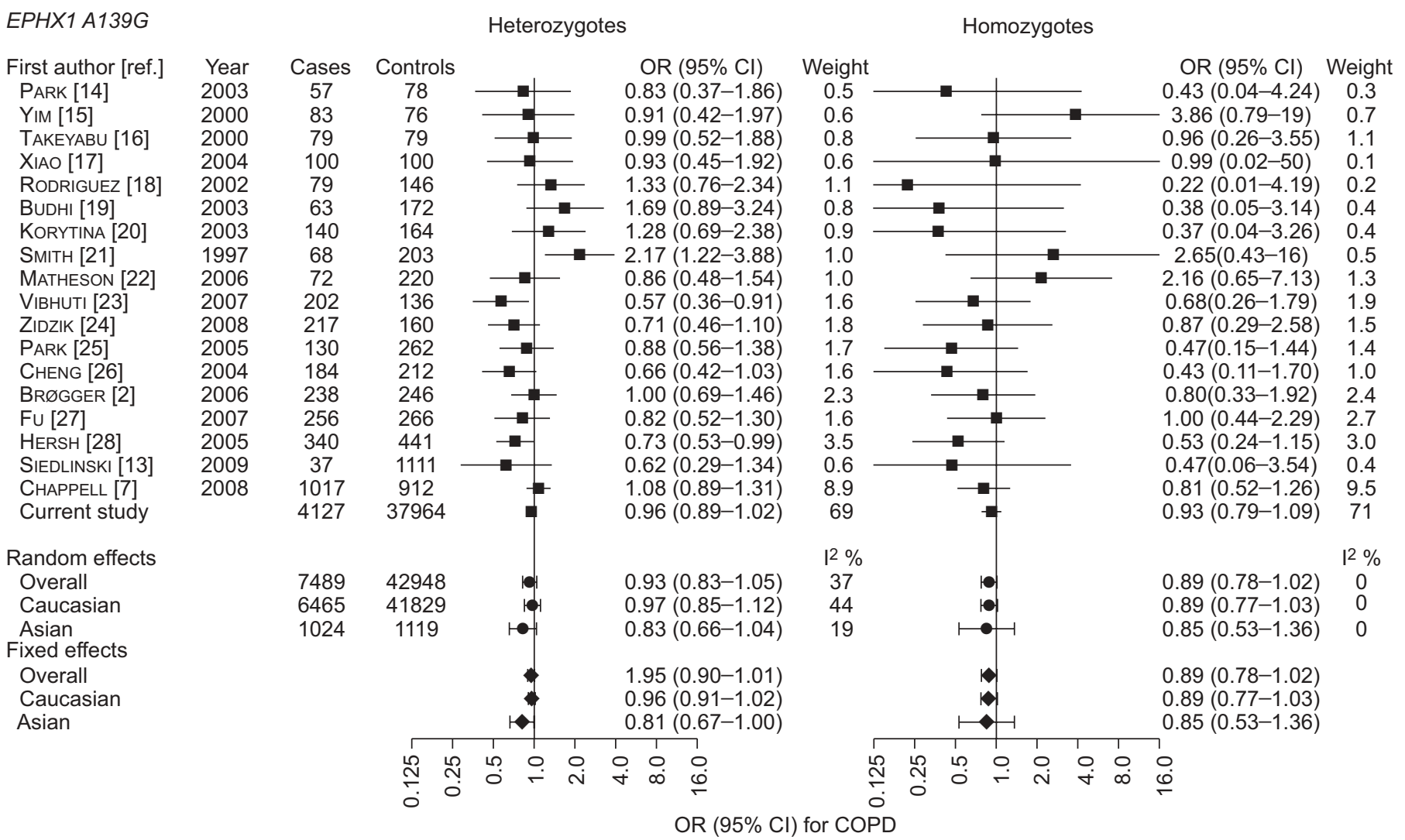

FIGURE 6. Meta-analysis of EPHX1 A139G genotype and risk of chronic obstructive pulmonary disease (COPD); overall and stratified analyses using both random and fixed effect models. Weights are from the fixed effect analyses using the inverse variance method. $\mathrm{I}^{2}$ : the variation in OR attributable to heterogeneity.

[11, 30-32], we have confidence in the negative findings of this study. However, the meta-analysis cannot completely exclude a minor effect of EPHX1 genotype on COPD risk.

\section{FEV $1 \%$ pred and decline in lung function}

Reports on the relationship between EPHX1 genotypes and phenotype and FEV1 as a main end-point in Caucasians are scarce and reach varying conclusions. One study found that the $113 \mathrm{C} / 139 \mathrm{~A}$ haplotype ( superslow phenotype) is associated with rapid decline in lung function [33]. In accordance with this, another study found that the putative fast allele, $139 G$ is protective against decline in lung function [28]. However, two recent studies, including the biggest study conducted prior to the present, find no impact of EPHX1 on FEV1 or decline in FEV1 with time $[13,22]$. Our results likewise do not support an association of EPHX1 with FEV1 or decline in FEV1.

\section{COPD}

Several reports exist linking EPHX1 polymorphisms to altered risk of COPD. Unfortunately, the picture is obscured by lack of consistency in the findings reported. Thus, studies investigating the individual polymorphisms variably find the $T 113 \mathrm{C}$ variant to be associated with either increased risk of [21] or protection from [2] COPD. Some suggest that progression rather than susceptibility to COPD is affected by the presence of the $113 \mathrm{C}$ allele [34]. One study found an association of the $113 \mathrm{C}$ allele with functional impairment in COPD [35]. Some studies indicate a protective effect of the putative fast $139 \mathrm{G}$ allele [1, 28]; however, many studies find no effect at all of either polymorphism [7, 22, 36]. Similar disparities are found in studies investigating association of COPD with EPHX1 phenotype $[7,21,22,36]$. Overall, there is a tendency that the effect of individual genotypes is observed mostly in Asians, whereas Caucasians are not affected [7, 22, 36, 37]. In concordance with this, our results indicate that EPHX1 genotype or phenotype is not associated with COPD in the Danish population.

Our main study did not reach statistical significance for the EPHX1 genotypes while the meta-analysis did for T113C homozygotes; however, the $95 \%$ CIs overlap, and so these results cannot be ruled contradictory. In accordance with our findings in the main study, meta-analysis did not indicate a significant effect of the $A 139 G$ variant allele. Even though statistical significance was not reached, there is a trend towards a lowered risk of COPD for $A 139 G$ variant allele carriers. This is consistent with the putatively increased microsomal epoxide hydrolase detoxifying activity associated with this allele. Likewise, the putatively lowered enzyme activity associated with the T113C variant allele corresponds well with the increased risk of COPD observed for this allele. Previous meta-analyses by Hu et al. [36] and BRøGGER et al. [2] are in agreement with our findings reporting a slightly increased risk of COPD for 113C homozygotes but no effect of the other genotypes. The most recent meta-analysis by SMOLONSKA et al. [37] investigates the effect of variant allele carriers versus noncarriers. They do not find any effect on the risk of COPD for either the $T 113 C$ or the $A 139 G$ polymorphisms. 


\section{Asthma}

Although the clinical manifestations of asthma and COPD are different, some of the same environmental factors may trigger both disease states $[6,38]$. Genetic variations in xenobioticmetabolising enzymes such as EPHX1 have been found to modify asthma susceptibility [39, 40]. SALAM et al. [40] report that high activity phenotypes of EPHX1 predisposes for asthma. This is in contrast to the findings of DUAN et al. [41] who hypothesise that EPHX1 may have a dual effect on susceptibility to asthma. They report that apart from lowering enzyme activity, the T113C variant also upregulates another gene (ORMDL3), which has been associated with risk of childhood asthma [42]. A recent pilot study also emphasises the multifactorial nature of bronchial asthma, reporting that gene-gene and gene-environment interactions involving EPHX1 are important determinants of asthma susceptibility [38]. We were not able to evaluate as many interactions as were in that study. However, our results point to the conclusion that the investigated polymorphisms of the EPHX1 gene are not major factors in susceptibility to asthma.

\section{Limitations}

The size of the effect of EPHX1 variants on risk of COPD may depend on ethnicity [36, 37]. All participants in the present study were white, Danish subjects, and while this eliminates any blurring due to ethnical heterogeneity of the study population, our results may apply to white subjects only. Our aim was to investigate polymorphisms of the EPHX1 gene in relation to COPD and other pulmonary outcomes overall. Although we did not find any such association, we cannot rule out the possibility that an association exists in certain subgroups of individuals.

As previously mentioned, the two functional SNPs in exons 3 and 4 , respectively, tag the entire coding region of the gene. However, no linkage disequilibrium is observed between the coding region and the promoter region [12]. Therefore, while our results suggest that the two functional SNPs and the corresponding haplotype blocks are not associated with risk of COPD or asthma, nor with level or decrease in lung function, we cannot exclude that variants in the promoter region of the EPHX1 gene may be associated with these traits.

\section{Summary}

Previous studies indicate that common polymorphisms in the EPHX1 gene are associated with altered risk of COPD and other pulmonary outcomes. Although we have studied the two polymorphisms with the highest predicted effect on microsomal epoxide hydrolase activity in humans, we found no consistent association between these polymorphisms and COPD, COPD-related traits, or asthma. Our results thus indicate with substantial statistical power that genetically reduced microsomal epoxide hydrolase activity per se is not a major risk factor for COPD or asthma in the Danish population. However, the meta-analysis cannot completely exclude a minor effect of EPHX1 genotype on COPD risk.

\section{STATEMENT OF INTEREST}

None declared.

\section{ACKNOWLEDGEMENTS}

This work was supported by The European Respiratory Society, The Danish Lung Foundation, The Danish Heart Association, The Capital
Region of Denmark Research Foundation, Chief Physician Johan Boserup and Lise Boserup's Fund, and Herlev Hospital, Copenhagen University Hospital.

\section{REFERENCES}

1 DeMeo DL, Hersh CP, Hoffman EA, et al. Genetic determinants of emphysema distribution in the national emphysema treatment trial. Am J Respir Crit Care Med 2007; 176: 42-48.

2 Brøgger J, Steen VM, Eiken HG, et al. Genetic association between COPD and polymorphisms in TNF, ADRB2 and EPHX1. Eur Respir J 2006; 27: 682-688.

3 Lokke A, Lange P, Scharling H, et al. Developing COPD: a 25-year follow up study of the general population. Thorax 2006; 61: 935-939.

4 Silverman EK, Chapman HA, Drazen JM, et al. Genetic epidemiology of severe, early-onset chronic obstructive pulmonary disease. Risk to relatives for airflow obstruction and chronic bronchitis. Am J Respir Crit Care Med 1998; 157: 1770-1778.

5 Foreman MG, DeMeo DL, Hersh CP, et al. Clinical determinants of exacerbations in severe, early-onset COPD. Eur Respir J 2007; 30: 1124-1130.

6 Tomaki M, Sugiura H, Koarai A, et al. Decreased expression of antioxidant enzymes and increased expression of chemokines in COPD lung. Pulm Pharmacol Ther 2007; 20: 596-605.

7 Chappell S, Daly L, Morgan K, et al. Genetic variants of microsomal epoxide hydrolase and glutamate-cysteine ligase in COPD. Eur Respir J 2008; 32: 931-937.

8 Hassett C, Aicher L, Sidhu JS, et al. Human microsomal epoxide hydrolase: genetic polymorphism and functional expression in vitro of amino acid variants. Hum Mol Genet 1994; 3: 421-428.

9 Schnohr P, Jensen JS, Scharling H, et al. Coronary heart disease risk factors ranked by importance for the individual and community. A 21-year follow-up of 12,000 men and women from The Copenhagen City Heart Study. Eur Heart J 2002; 23: 620-626.

10 Nordestgaard BG, Benn M, Schnohr P, et al. Nonfasting triglycerides and risk of myocardial infarction, ischemic heart disease, and death in men and women. JAMA 2007; 298: 299-308.

11 Dahl M, Bowler RP, Juul K, et al. Superoxide dismutase 3 polymorphism associated with reduced lung function in two large populations. Am J Respir Crit Care Med 2008; 178: 906-912.

12 Yang X, Liang SH, Weyant DM, et al. The expression of human microsomal epoxide hydrolase is predominantly driven by a genetically polymorphic far upstream promoter. J Pharmacol Exp Ther 2009; 330: 23-30.

13 Siedlinski M, Postma DS, Smit HA, et al. No effects of EPHX1 polymorphisms on the level or change of FEV1 in the general population. Eur Respir J 2009; 33: 446-449.

14 Park SS, Kim EJ, Son CY, et al. Genetic polymorphism of epoxide hydrolase and GSTM1 in chronic obstructive pulmonary disease. Tuberc Respir Dis 2003; 55: 88-97.

15 Yim JJ, Park GY, Lee CT, et al. Genetic susceptibility to chronic obstructive pulmonary disease in Koreans: combined analysis of polymorphic genotypes for microsomal epoxide hydrolase and glutathione S-transferase M1 and T1. Thorax 2000; 55: 121-125.

16 Takeyabu K, Yamaguchi E, Suzuki I, et al. Gene polymorphism for microsomal epoxide hydrolase and susceptibility to emphysema in a Japanese population. Eur Respir J 2000; 15: 891-894.

17 Xiao D, Wang C, Du MJ, et al. Relationship between polymorphisms of genes encoding microsomal epoxide hydrolase and glutathione $S$-transferase P1 and chronic obstructive pulmonary disease. Chin Med J (Engl) 2004; 117: 661-667.

18 Rodriguez F, Jardi R, Costa X, et al. Detection of polymorphisms at exons 3 (Tyr113--> His) and 4 (His139-->Arg) of the microsomal epoxide hydrolase gene using fluorescence PCR method combined with melting curves analysis. Anal Biochem 2002; 308: 120-126. 
19 Budhi A, Hiyama K, Isobe T, et al. Genetic susceptibility for emphysematous changes of the lung in Japanese. Int J Mol Med 2003; 11: 321-329.

20 Korytina GF, Yanbaeva DG, Viktorova TV. Polymorphisms of the cytochrome P450 (CYP1A1, CYP2E1) and microsomal epoxide hydrolase (mEPHX) genes in cystic fibrosis and chronic respiratory disease. Mol Biol 2003; 37: 663-670.

21 Smith CA, Harrison DJ. Association between polymorphism in gene for microsomal epoxide hydrolase and susceptibility to emphysema. Lancet 1997; 350: 630-633.

22 Matheson MC, Raven J, Walters EH, et al. Microsomal epoxide hydrolase is not associated with COPD in a community-based sample. Hum Biol 2006; 78: 705-717.

23 Vibhuti A, Arif E, Deepak D, et al. Genetic polymorphisms of GSTP1 and mEPHX correlate with oxidative stress markers and lung function in COPD. Biochem Biophys Res Commun 2007; 359: 136-142.

24 Zidzik J, Slaba E, Joppa P, et al. Glutathione S-transferase and microsomal epoxide hydrolase gene polymorphisms and risk of chronic obstructive pulmonary disease in Slovak population. Croat Med J 2008; 49: 182-191.

25 Park JY, Chen L, Wadhwa N, et al. Polymorphisms for microsomal epoxide hydrolase and genetic susceptibility to COPD. Int J Mol Med 2005; 15: 443-448.

26 Cheng SL, Yu CJ, Chen CJ, et al. Genetic polymorphism of epoxide hydrolase and glutathione S-transferase in COPD. Eur Respir J 2004; 23: 818-824.

27 Fu WP, Sun C, Dai LM, et al. Relationship between COPD and polymorphisms of HOX-1 and $\mathrm{mEPH}$ in a Chinese population. Oncol Rep 2007; 17: 483-488.

28 Hersh CP, DeMeo DL, Lange C et al. Attempted replication of reported chronic obstructive pulmonary disease candidate gene associations. Am J Respir Cell Mol Biol 2005; 33: 71-78.

29 Keicho N, Emi M, Kajita M, et al. Overestimated frequency of a possible emphysema-susceptibility allele when microsomal epoxide hydrolase is genotyped by the conventional polymerase chain reaction-based method. J Hum Genet 2001; 46: 96-98.

30 Juul K, Tybjaerg-Hansen A, Marklund S, et al. Genetically increased antioxidative protection and decreased chronic obstructive pulmonary disease. Am J Respir Crit Care Med 2006; 173: 858-864.
31 Dahl M, Tybjaerg-Hansen A, Lange $\mathrm{P}$, et al. Change in lung function and morbidity from chronic obstructive pulmonary disease in $\alpha_{1}$-antitrypsin MZ heterozygotes: A longitudinal study of the general population. Ann Intern Med 2002; 136: 270-279.

32 Baekvad-Hansen M, Dahl M, Tybjaerg-Hansen A, et al. Surfactant protein-B 121ins2 heterozygosity, reduced pulmonary function, and chronic obstructive pulmonary disease in smokers. Am J Respir Crit Care Med 2010; 181: 17-20.

33 Sandford AJ, Chagani T, Weir TD, et al. Susceptibility genes for rapid decline of lung function in the lung health study. Am J Respir Crit Care Med 2001; 163: 469-473.

34 Yoshikawa M, Hiyama K, Ishioka S, et al. Microsomal epoxide hydrolase genotypes and chronic obstructive pulmonary disease in Japanese. Int J Mol Med 2000; 5: 49-53.

35 Hersh CP, DeMeo DL, Lazarus R, et al. Genetic association analysis of functional impairment in chronic obstructive pulmonary disease. Am J Respir Crit Care Med 2006; 173: 977-984.

$36 \mathrm{Hu} \mathrm{G}$, Shi Z, Hu J, et al. Association between polymorphisms of microsomal epoxide hydrolase and COPD: results from metaanalyses. Respirology 2008; 13: 837-850.

37 Smolonska J, Wijmenga C, Postma DS, et al. Meta-analyses on suspected chronic obstructive pulmonary disease genes: a summary of 20 years' research. Am J Respir Crit Care Med 2009; 180: 618-631.

38 Polonikov AV, Ivanov VP, Solodilova MA. Genetic variation of genes for xenobiotic-metabolizing enzymes and risk of bronchial asthma: the importance of gene-gene and gene-environment interactions for disease susceptibility. J Hum Genet 2009; 54: 440-449.

39 Moffatt MF. Genes in asthma: new genes and new ways. Curr Opin Allergy Clin Immunol 2008; 8: 411-417.

40 Salam MT, Lin PC, Avol EL, et al. Microsomal epoxide hydrolase, glutathione $S$-transferase P1, traffic and childhood asthma. Thorax 2007; 62: 1050-1057.

41 Duan S, Huang RS, Zhang W, et al. Genetic architecture of transcript-level variation in humans. Am J Hum Genet 2008; 82: 1101-1113.

42 Moffatt MF, Kabesch M, Liang L, et al. Genetic variants regulating ORMDL3 expression contribute to the risk of childhood asthma. Nature 2007; 448: 470-473. 